\title{
Photoluminescence from gold nanoparticles modified by polyvinylpyrrolidone (PVP) via hydrothermal synthesis
}

\author{
Weilin Du ${ }^{1, a}$, Li Jiang ${ }^{1, b}$, Lei Liao ${ }^{2, c}$, Aimiao Qin ${ }^{1,3 d^{*}}$, and Shuoping Chen ${ }^{1, e}$ \\ ${ }^{1}$ Key Lab New Processing Technology for Nonferrous Metals \& Materials Ministry of \\ Education, Guilin University of Technology, No.12 Jiangan Road, Guilin, 541004, P. R. China \\ ${ }^{2}$ College of Environmental Science and Engineering, Guilin University of Technology, No.12 \\ Jiangan Road, Guilin, 541004, P. R. China \\ ${ }^{3}$ Guangxi Experiment Center of Information Science, Guilin, 541004, P. R. China

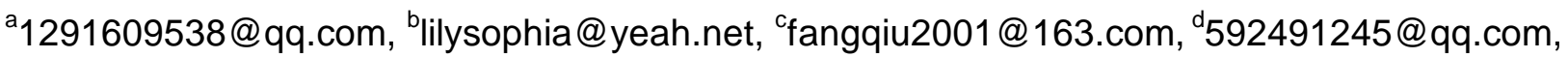 \\ 277087163@qq.com \\ ${ }^{*}$ Corresponding author
}

Keywords: Gold nanoparticles, PVP, photoluminescence spectra, optical materials, hydrothermal synthesis

Abstract. Luminescent gold nanoparticles(NPs) modified by Polyvinylpyrrolidone (PVP) with high stability and well dispersed property were successfully via synthesized hydrothermal method. UV-visible spectrophotometer, fluorescence spectrophotometer, fourier transform infrared spectrophotometer (FT-IR) and transmission electron microscopy (TEM) were used to study the influence of hydrothermal reaction conditions such as the reaction time, reaction temperature and dosage of PVP on the luminescent properties of colloidal gold solution. We found that the relative luminescence intensity of colloidal gold solution increases with the increasing of reaction time, reaction temperature and dosage of PVP, which is partly due to the increase of crystallinity. We also found that PVP plays a key role in the formation of luminescent AuNPs and a possible mechanism for the formation of luminescent AuNPs was put forward.

\section{Introduction}

Gold nanoparticles (AuNPs) have shown favorable stability, biocompatibility, high density and unique optical properties and have potential applications in catalysis, biolabel, photonics, optoelectronics and so on [1]. In 1968, Mooradian[2] reported that a faint photoluminescence ( was observed from gold metals, which attracted considerable interests in the photoluminescence of noble metals. Literature research shows that strong photoluminescence from gold metals can be observed when their size decrease to the nano-order. Luminescent Au nanoclusters often have size smaller than $2 \mathrm{~nm}$ in aqueous and usually have a protective layer, Glutathione, tiopronin, and dentritic structures of PAMAM often were use as the molecular layer. PVP is a kind of polymer surfactant and easily soluble in water but less toxicity and better biocompatibility [3]. Besides served as surfactant or stabilizers in the preparation of nanostructures, a couple of papers mentioned that PVP could be served as reducing agent[4-5]. For example, Xuefeng Qian and co-workers used PVP as the stabilizer and weaker reducing agent for synthesizing Ag nanoparticles under microwave irradiation [6]. However, as far as we know, there are very few reports on the investigation of the photoluminescence properties of AuNPs with size larger than 5nm in the presence of PVP. In this paper, AuNPs were prepared by a PVP-assisted hydrothermal method from an aqueous solution of chloroauric acid tetrahydrate $\left(\mathrm{HAuCl}_{4} \cdot 4 \mathrm{H}_{2} \mathrm{O}\right)$. The influences of preparation conditions on the optical properties especially the photoluminescence properties of gold colloids were investigated in detail, including the different reaction time, different reaction temperature and the dosage of PVP. The possible mechanism formation of luminescent AuNPs was also discussed. 


\section{Experimental Section}

Instrumentation Solution samples for ultraviolet-visible (UV-vis) spectra and photoluminescence (PL) spectra were used as the prepared without diluting or enrichment. The UV-vis spectra were taken from TU-1901 double beam UV-vis spectrophotometer and the PL were carried out on a VARIAN fluorescence spectrophotometer. The molecular structures of PVP were characterized by AVATAR360 fourier transform infrared (FT-IR) spectrophotometer. Both the size and the shape of AuPNs samples were characterized by JSM-6380LV transmission electron microscope (TEM).

Preparation gold colloids All of chemicals used for the synthesis were of analytical grade and used as received without any further processing. $\mathrm{HAuCl}_{4} \cdot 4 \mathrm{H}_{2} \mathrm{O}$ was purchased from Sinopharm Chemical Reagent Co., Ltd. PVP (K-30) with average molecular weight of $40 \mathrm{k}$ was purchased from the Chinese medicine group chemical reagent limited company.

In a typical procedure, $4.9 \times 10^{-6} \mathrm{~mol} \mathrm{PVP}$ and $4.9 \times 10^{-7} \mathrm{~mol} \mathrm{HAuCl}_{4} \cdot 4 \mathrm{H}_{2} \mathrm{O}$ were dissolved in $32 \mathrm{ml}$ distilled water under constant magnetic stirring at room temperature to obtain a homogeneous reaction mixture. Then the above mixture was transferred into a $40 \mathrm{ml}$ Teflon-lined stainless steel autoclave and heated to a fixed temperature $\left(80-160{ }^{\circ} \mathrm{C}\right.$ ) for $2-12 \mathrm{~h}$, and then was allowed to cool to room temperature naturally. The obtained colloidal solutions with colors were stored for characterization without dilution.

\section{Results and discussion}

Figure 1 shows the UV-vis absorbance and PL spectra(A) and TEM image (B) of the typical pink colloidal Au solution prepared with a molar ratio of $\mathrm{HAuCl}_{4} \cdot 4 \mathrm{H}_{2} \mathrm{O}: \mathrm{PVP}=1: 10$, at the temperature of $120{ }^{\circ} \mathrm{C}$ for 8 hours. From Fig. 1 (A), it can be seen that the maximum absorbance peak of colloidal $\mathrm{Au}$ solution locates at $530 \mathrm{~nm}$. It is well known that the optical properties such as absorption maxima and absorption intensity are particle size dependent, an intense absorption peak at $530 \mathrm{~nm}$ generally attributed to the surface plasmon excitation of small spherical gold particles with size less than 50nm. The PL spectrum of the colloidal AuNPs measured with an excitation wavelength of $308 \mathrm{~nm}$ is also displayed in Fig. 1 (A). The centre of the PL band appears at $410 \mathrm{~nm}$ with a full width half maximum (FWHM) of $70 \mathrm{~nm}$. To confirm the peak appears in PL spectrum is from AuNPs but not from PVP molecules, a series of experiments were carried out. Firstly, both of the PL spectra of PVP solution before and after hydrothermal treatment were tested. The emission peak position of the both solution was also found near $410 \mathrm{~nm}$, but the intensity was much lower than that of AuNPs in the presence of PVP(Fig.2 A). The emission intensity order is as following: AuNPs in the presence of PVP » $\mathrm{PVP}$ with hydrothermal treatment $>$ PVP. Secondly, kept the other experimental parameters the same, we used PEG(MW6000) to replace PVP, PL peak at $440 \mathrm{~nm}$ of AuNPs in the presence of PEG was observed, but it disappeared for the pure PEG solution before and after hydrothermal treatment (Fig.2 B). Herein, we can claim that the PL peak of Au colloids is induced by Au nanoparticles. It is interesting to find that the PL peak of Au colloids appears only in the presence of polymer. According to the literature, metallic particles do not normally exhibit PL, however, PL can be observed from "non-metallic"Au clusters with very low nuclearity, which is well coincident to our experiments results.

The TEM image (Fig. 1 B) reveals the morphology of the prepared AuNPs in the presence of PVP, where AuNPs are well mono-dispersed, homogeneous and spherical shape with a diameter about 8-10 nm, some small AuNPs with a diameter less than $1.5 \mathrm{~nm}$ were also observed. 

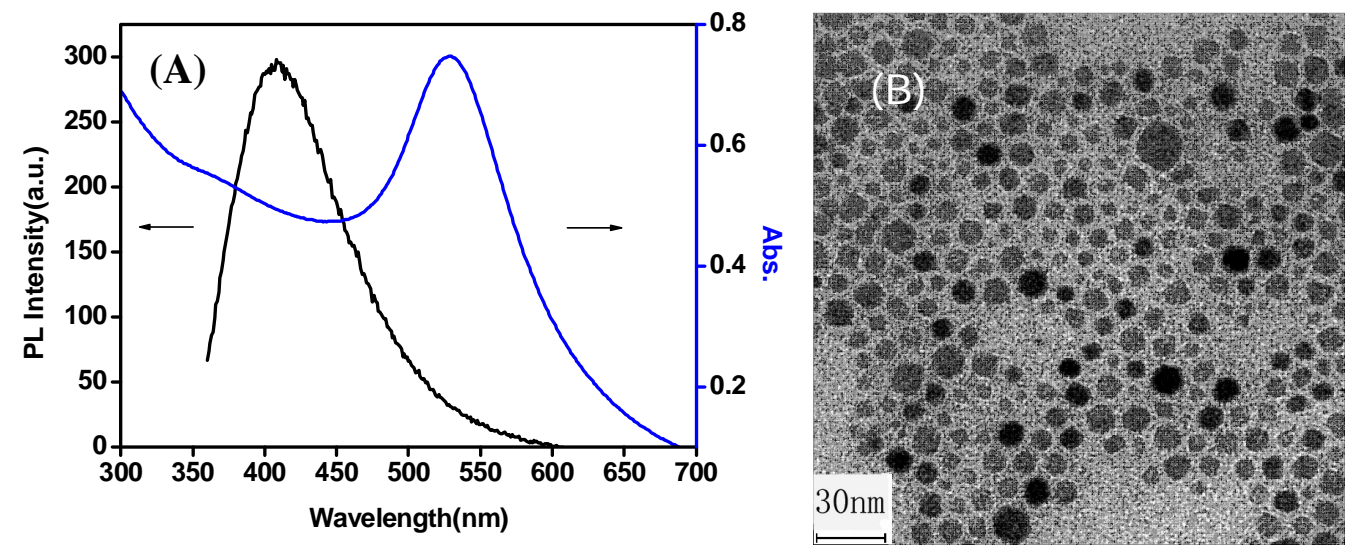

Fig.1. (A) The UV-vis and PL spectrum and (B) TEM image of the typical colloidal gold solution
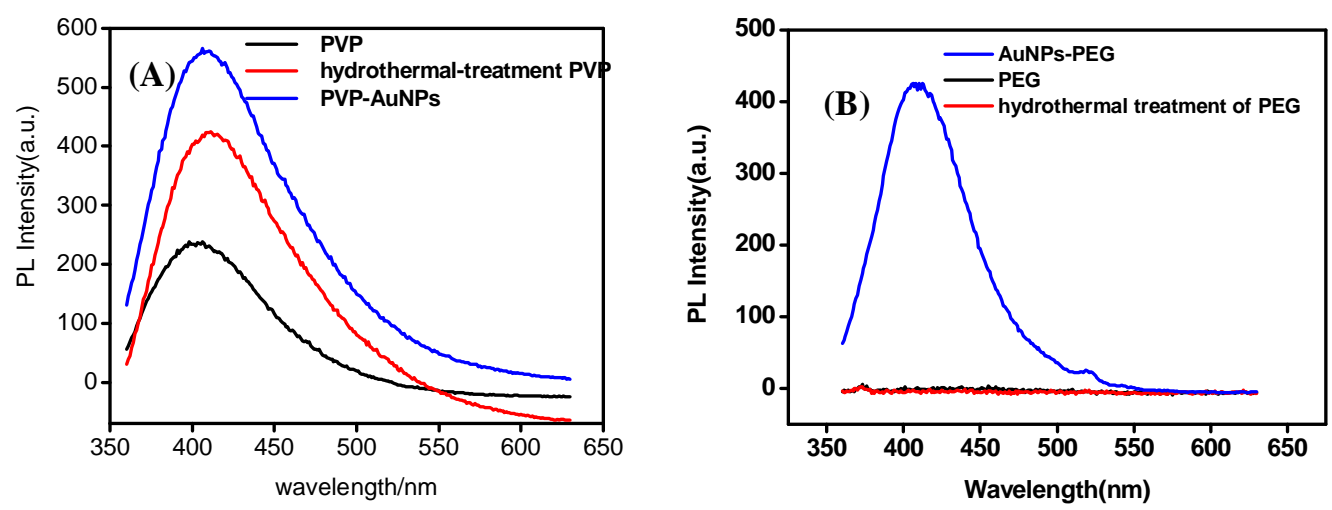

Fig.2. The PL spectra of AuNPs colloid in the presence of PVP (A) and (B) PEG

The UV-vis and PL spectra of colloidal Au solution in the presence of PVP prepared at different hydrothermal temperatures from $80-160^{\circ} \mathrm{C}$ show that the intensity of the maximum absorbance peak of colloidal $\mathrm{Au}$ solution varied with the increase of the temperature, firstly increased and then decreased, it reached to a maximum at the temperature of $100^{\circ} \mathrm{C}$, but decreased higher than $120^{\circ} \mathrm{C}$. The maximum absorbance peak at $530 \mathrm{~nm}$ became broadened with the increase temperature and it seems nearly to disappear at $160^{\circ} \mathrm{C}$, which indicated the size change of Au particles. The PL emission peak increased with the rise of the temperature and a small shift of the peak was obvious. The color of $\mathrm{Au}$ solution changed with the increasing temperature, from pink $\left(80-120{ }^{\circ} \mathrm{C}\right)$ - light yellow $\left(140{ }^{\circ} \mathrm{C}\right)$ to soil color $\left(160{ }^{\circ} \mathrm{C}\right)$, which also indicated the size of Au had changed. TEM images of AuNPs prepared at different temperature from $80-160^{\circ} \mathrm{C}$ obviously showed that the congestion appears when the temperature higher than $140^{\circ} \mathrm{C}$, the size of AuPNs and the crystallinity increased with the temperature, the average size of AuPNs prepared at $80{ }^{\circ} \mathrm{C}, 100^{\circ} \mathrm{C}, 140^{\circ} \mathrm{C}$ and $160{ }^{\circ} \mathrm{C}$ was about 3 $\mathrm{nm}, 10 \mathrm{~nm}, 12 \mathrm{~nm}$ and $11.4 \mathrm{~nm}$, respectively. The aggregation appeared when temperature higher than $140^{\circ} \mathrm{C}$ maybe due to the entanglement of PVP molecular chains. It is well-known that the PL of Quantum dots(QDs) is intensively affected by the environment, such as temperature, solvents, $\mathrm{pH}$, surfactants and particle sizes, different interparticle distance, surface defects and etc. The intensity of colloidal Au solution in the presence of PVP increased with the rise of the reaction temperature can be explained by the crystallinity increase of Au and the enhanced of PVP.

The UV-vis absorbance spectra and PL spectra of AuPNs in the presence of PVP recorded in a function of hydrothermal time of reaction showed that all the color of the solutionwas pink and the emission spectra and excitation spectra were completely symmetrical in the range of reaction time 2 $12 \mathrm{~h}$. The intensity of UV-vis absorbance peaks firstly increased with the extension of reaction time, then decreased after $12 \mathrm{~h}$, it reached to maximum at $8 \mathrm{~h}$. Which indicated that the reaction had not completed if the reaction time is less than $8 \mathrm{~h}$, but when is extended to longer than $12 \mathrm{~h}$, the agglomeration would happen. Both of the emission and excitation PL intensity of colloidal Au solution 
increased with the extension of reaction time. TEM observation showed that the size of AuNPs increased from 2 to $10 \mathrm{~nm}$ with the extension of reaction time from 2 to $12 \mathrm{~h}$.

The study of the effects of PVP dosage on the UV-vis and PL spectra of AuNPs solution showed that the absorption intensity of AuNPs in the presence of PVP (PVP-AuNPs) varied without any regulations when the molecular ratio of PVP to $\mathrm{HAuCl}_{4}$ increased in the range from 2.5:1 to 7.5: 1, however, it increases with the increase of the molecular ratio of PVP to $\mathrm{HAuCl}_{4}$ from $10: 1$ to $40: 1$. The PL intensity of both PVP-AuNPs and PVP solution linearly increased with the increasing of the dosage of PVP. A possible mechanism for luminescent AuNPs in the presence of PVP is described as, firstly, $\mathrm{AuCl}_{4}{ }^{-}$ions was reduced to $\mathrm{Au}$ by nitrogen atom of five-membered heterocyclic structure of PVP in the hydrothermal condition and some combine together and then grow up to Au clusters. Then, some PVP molecules with the function of capped ligand for Au clusters formed "non-metallic"Au clusters and exhibited PL. The capped ligand of PVP decreased the number of surface defects and increased the robustness of the NCs at the same time, which increased the PL intensity of Au NCs. Furthermore, PVP molecules were dissolved in aqueous solution and formed micelles to enhance the PL of AuNPs. Therefore, luminescent AuNPs with size in uniformity and spherical morphology finally performed.

\section{Conclusions}

In conclusion, luminescent gold colloid was successfully prepared by hydrothermal method using $\mathrm{HAuCl}_{4}$ and PVP as precursors. It showed that reaction temperature and reaction time had much effect on the PL and UV-vis spectra. The PL intensity increased with the rise of reaction temperature, reaction time and the dosage of PVP. The enhanced fluorescence of gold colloid was largely due to the properties of PVP. These results contribute to understand the fluorescent mechanism of AuNPs and expected to be used in biological detection, drug release, etc.

\section{Acknowledgments}

This work was supported by the National Science Foundation of China (No: 21063005,51564009 and 51468011), the National Science Foundation of Guangxi Province(No.2015GXNSFDA139035) and by the Foundation of Guangxi Experiment Center of Information Science (No: KF1407).

\section{References}

[1] Y. N. Xia, W. Y. Li, C. M. Cobley, J. Y. Chen, X. H. Xia, Q. Zhang, M. X. Yang, E. C. Cho, P. K. Brown, Gold nanocages: from synthesis to theranostic applications, Acc. Chem. Res. 44 (2011) 914-924.

[2] A. Mooradian, Photoluminescence of Metals, Phys. Rev. Lett., 22(1969)185 - 187.

[3] K. Kumagai, A. Ishida. Synthesis and growth observation of flower- and spike-like gold-silver nanoparticles, Chem. Lett. 41(2012)580-582.

[4] Y. G. Sun, Y. N. Xia, Large-scale synthesis of uniform silver nanowires through a soft, self-seeding, polyol process, Adv. Mater. 14 (2002) 833-837

[5] M. Tsuji, M. Hashimoto, Y. Nishizawa, M. Kubokawa, and T. Tsuji, Microwave-assisted synthesis of metallic nanostructures in solution, Chem. Eur. J. 2005, 11, $440-452$.

[6] He R, Qian X F, Yin J, Zhu Z K, Preparation of polychrome silver nanoparticles in different solvents. J. Mater. Chem., 12(2002) 3783-3786.

[7] R. Tarnawski, M. Ulbricht, Amphiphilic gold nanoparticles: Synthesis, characterization and adsorption to PEGylated polymer surfaces. Colloids and Surfaces A: Physicochem. Eng. Aspects 374 (2011) 13-21.

[8] X. D. Cheng, X. B. WANG. Fluorescence Spectra of Poly ( vinylpyrrolydone) in Aqueous Solution, Acta Scientiarum Naturalium Universitatis Sunyatseni. 43(2004) 54-57. 